\title{
MCMC for Wind Power Simulation
}

\author{
George Papaefthymiou, Member, IEEE, and Bernd Klöckl, Member, IEEE
}

\begin{abstract}
This paper contributes a Markov chain Monte Carlo (MCMC) method for the direct generation of synthetic time series of wind power output. It is shown that obtaining a stochastic model directly in the wind power domain leads to reduced number of states and to lower order of the Markov chain at equal power data resolution. The estimation quality of the stochastic model is positively influenced since in the power domain, a lower number of independent parameters is estimated from a given amount of recorded data. The simulation results prove that this method offers excellent fit for both the probability density function and the autocorrelation function of the generated wind power time series. The method is a first step toward simple stochastic black-box models for wind generation.
\end{abstract}

Index Terms-Monte Carlo simulation, Markov chain, wind energy conversion, wind turbine generator.

\section{INTRODUCTION}

W IND ENERGY has been the fastest growing energy technology in the last years. In spite of its indisputable benefits, the increasing share of wind generation in the power system energy mix can bring unwanted consequences, mainly due to the stochastic nature of wind power. In order to study the impact of wind generation to the power system, time series of the power output of the wind plants are used, usually obtained by a procedure consisting of two distinct steps: the utilization of data and information on the stochastic prime mover (wind activity) and the subsequent transformation of the wind activity to power output.

In order to capture the wind stochasticity, recordings for a sufficiently long period should be used. However, because the data records typically are of short length, it can become necessary to use stochastic simulation techniques for the derivation of synthetic time series for the modeling of the wind activity. The direct and indiscriminate use of short measured time series as input to the subsequent modeling procedure may lead to wrong conclusions on system design issues, especially when the records are short with respect to the time constants of processes within the studied system. A typical example is the dimensioning of a combined wind power/energy storage plant with relatively high

Manuscript received July 12, 2006; revised March 27, 2007. This work was supported in part by SenterNovem (Intelligent Power Systems Project Web site [Online]. Available: http://www.intelligentpowersystems.nl, 2003), an agency of the Dutch ministry of Economic Affairs, in part by the $\mathrm{ABB}$, Areva, Siemens, and in part by the Swiss Federal Office for Energy (The Vision of Future Energy Networks Web site [Online]. Available: http://www.eeh.ee.ethz.ch/hvl/research/vofen.html.) Paper no. TEC-002822006.

G. Papaefthymiou is with the Electric Power Systems Group, Delft University of Technology, Delft $2628 \mathrm{CD}$, The Netherlands (e-mail: g.papaefthymiou@tudelft.nl).

B. Klöckl was with the High Voltage Laboratory, Swiss Federal Institute of Technology (ETH), Zurich CH-8092, Switzerland. He is now with Verbund Austrian Power Grid, A-1220 Vienna, Austria (e-mail: Bernd.kloeckl@verbund.at).

Digital Object Identifier 10.1109/TEC.2007.914174 energy capacity. Simple simulation investigations show that for energy capacities of more than $24 \mathrm{~h}$ of nominal power, even one year of measured high-quality data can be entirely insufficient to estimate the statistical properties of the stored energy content. The reason is that the stochastic process "energy storage" must by theoretical considerations be more persistent than the stochastic process "energy storage terminal power," and hence, takes longer time to converge in the statistical sense than the generating stochastic process (e.g., the wind power time series). In other words, depending on the energy storage technology, one will have to use time series of different length for convergence of the result [1]. Similar effects can be observed in investigations on hybrid systems with relatively inert plants used for the compensation of stochastic sources, such as e.g., fuel cells. This fundamental problem of power system design in stochastically driven environments was recognized early by Klein and Beckman [2], who come to the conclusion that "... even 20 years of data are insufficient to obtain accurate estimates of loss of load probability $(L L P)$ for LLP values less than $0,01 \ldots$.." The necessity to at least have at hand a suitable method to simulate stochastic power time series from measured data becomes more urgent as systems to be designed based on stochastic sources become more complex, more densely meshed with a higher penetration of inert distributed resources to support the stochastic generation facilities such as storage, demand side management facilities, or backup generation.

Independent of the source of data, be it a natural stream of wind speed values, be it a synthetically processed data stream, the wind speed is typically the basis for the investigation. The second step in obtaining the wind power time series is the modeling of the energy conversion, i.e., the transformation of the wind activity (either measured or synthetic) into power. This refers to the modeling of the wind plant power curve and can be a complex procedure that should take into account the individual turbine curves, the terrain characteristics, the shadowing effects inside the wind farm, and the meteorological parameters' influence (e.g., ambient temperature, air density, turbulence intensity) on the production. Due to the complexity in deriving the detailed power curve, in most cases, a less accurate wind speed/wind power curve is used as the input-output characteristic. The output of this two-step procedure is a time series of the power fed to the grid connection point.

In this paper, we propose the development of a synthetic wind power model from measured data directly in the power domain and show that this procedure offers basic advantages over modeling procedures in the wind speed domain. We show that the aforementioned two-step modeling procedure may lead to an unnecessary increase in the complexity of the stochastic model, since the wind speed models contain information that is statistically redundant for the wind power model. We discuss the use of Markov chain Monte Carlo (MCMC) methods as an 


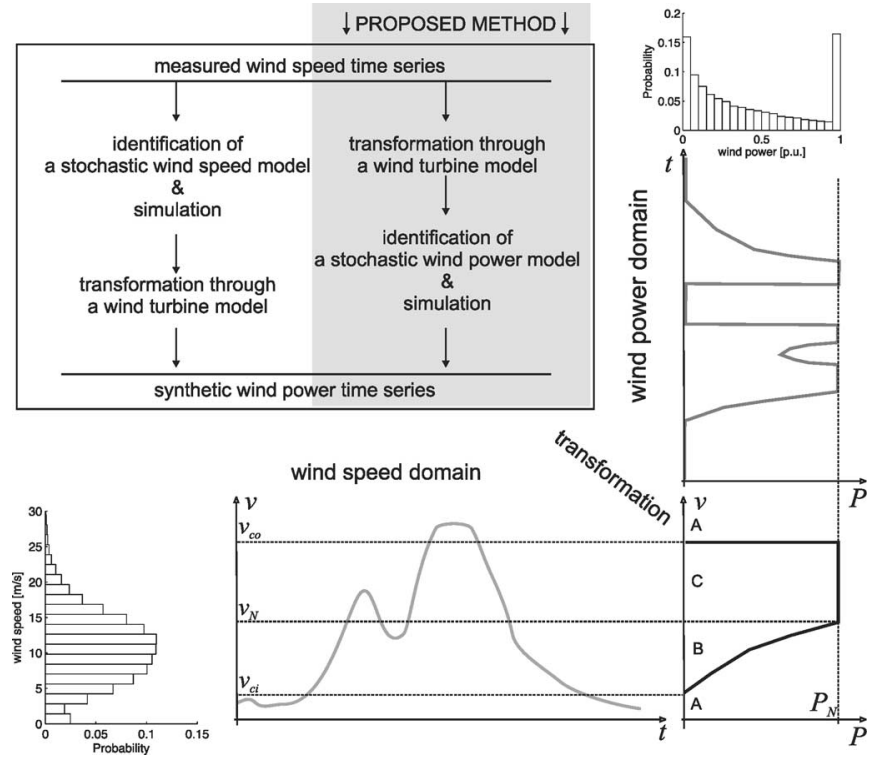

Fig. 1. Wind speed to wind power transformation through the nonlinear power curve of a single turbine. The boxed area shows the alternatives for a respective simulation algorithm.

appropriate representation of wind speed and power, and we compare the MCMC model in the wind speed and wind power domains and show that the wind power representation is more appropriate.

\section{StOchastic Models OF Wind POWER}

Stochastic models of the power output of a wind plant are more simple than the ones concerning wind activity, mainly due to the type of the transformation of the wind stochastic process into power. One can see this by the investigation of the wind speed/wind power curve of the wind turbine generator.

Wind turbine static power curves are nonlinear and transform the random variable (r.v.) "wind speed" $(v)$ into the r.v. "wind power" $[P(v)]$ according to the following equation

$$
P(v)=\left\{\begin{array}{lll}
0, & v \leq v_{c i} \text { or } v>v_{c o} & (\text { area } \mathrm{A}) \\
P_{N}, & v_{N} \leq v \leq v_{c o} & (\text { area } \mathrm{B}) \\
f(v), & v_{c i}<v<v_{N} & (\text { area } \mathrm{C})
\end{array}\right.
$$

where $v_{c i}, v_{c o}$, and $v_{N}$ are the cut-in, cut-out, and the nominal wind speed, respectively. $f(v)$ is a relation in the standard working range of the turbine dependent on the control strategy, aerodynamics, and the grid coupling technology. An illustration is given in Fig. 1. We can see that a mapping of the information between the wind speed and the wind power domain is achieved only in area $\mathrm{C}\left(v_{c i}<v<v_{N}\right)$, due to the monotonic coupling between the input and the output. In the other two areas, all wind speed values are mapped to one power value, either nominal (area B) or zero (area A). As may be seen in Fig. 1, this transformation of the wind speed distribution leads to an accumulation of probability masses to the power output distribution, corresponding to the nominal and zero output power areas [3]. A detailed wind speed representation contains unnecessary information for these two regions. In order to shift toward a simpler model of wind power, we propose the inversion of the modeling

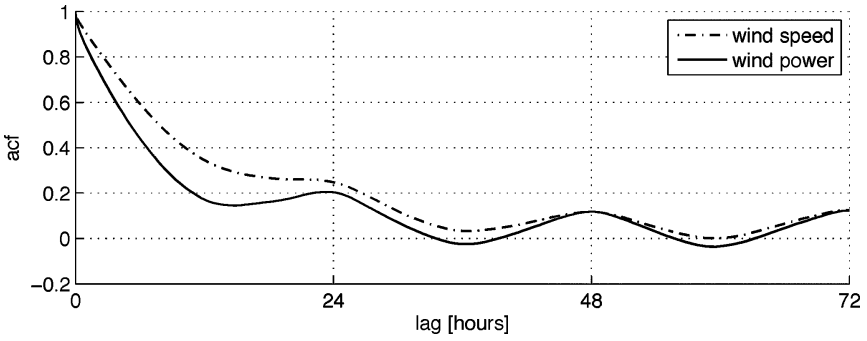

Fig. 2. acf obtained from measured wind speed and transformed wind power time series. The wind power time series shows lower persistence. The data were taken from TS A.

activity: instead of building a model in the wind speed domain, and then, transform to wind power, one should first transform to wind power, and then, obtain a wind power model directly in the wind power domain (Fig. 1, boxed area).

\section{MARKov ChAINS FOR THE DESCRIPTION OF WIND POWER TIME SERIES}

\section{A. Pdf and Acf of Wind Speed and Wind Power}

Methods for the synthetic generation of wind time series have to mimic the major statistical properties of the recorded data, namely the probability density function (pdf) and the autocorrelation function (acf) as closely as possible. As shown in Fig. 1, due to the impact of the nonlinear power curve, the wind speed pdf (approximated by a Weibull distribution) is transformed to a wind power distribution that presents accumulation of probability masses in the zero and nominal power values. The pdf is the statistical property associated to the wind energy output over longer periods (integration of the wind power pdf). The acf, namely the correlation of the stochastic process against a time-shifted version of itself, is a measure of the chronological persistence of the process. Wind speed time series show a relatively pronounced chronological persistence, imaged by a positive acf. The acf is also influenced by the transformation of $v$ through $P(v)$. In particular, in the zero and nominal power regions, consecutive wind speed observations become statistically independent in the wind power domain, which causes the empirical acf to be steeper for $P(v)$ than for $v$ (see, for example, Fig. 2). ${ }^{1}$

\section{B. Review of Related Work}

Two basic methodologies for the synthetic generation of time series may be found in the related literature: the classical timeseries analysis with autoregressive (AR) models and the MCMC techniques. Both methods present different advantages and disadvantages, making them suited for different applications.

Classical time-series analysis involves the generation of synthetic stationary time series based on the combination of the AR and moving average (MA) models for the stochastic process $[5] .^{2}$ The basic advantage of the methodology is that by

\footnotetext{
${ }^{1}$ For a good overview on the prominent statistical properties of wind with respect to wind power generation, see [4].

${ }^{2}$ For initial works on time-series analysis for wind speed sequences, see, e.g., [6] and [7].
} 
the definition of a relatively small number of parameters, the AR and ARMA models allow for controlled synthetization of a desired acf. This, however, does not hold for the pdf; in general, the pdf of the generated time series does not match the one of the measured data. Such a pdf mismatch can lead to wrong estimates of the energy yield of the process, and consequently, to wrong calculation of power flow densities with basic implications on system design.

For the synthetic generation of time series consistent with both basic statistical properties (i.e., pdf and acf), the MCMC method may be used instead. The application of the method involves the discretization of the stochastic process into a number of states and the definition of the probabilities for the transitions between states. Two are the basic disadvantages of the method, a possible loss of information due to the discretization procedure and the model complexity (a large number of parameters should be assessed from the recorded data, i.e., all probabilities for the mutual transitions between different system states). The definition of the number of states introduces a tradeoff between model accuracy and complexity; a higher discretization leads to a better representation of the process and also introduces a large number of parameters that are difficult to assess from data.

The MCMC literature on the modeling of wind speed and wind power focuses on developing the stochastic model in the wind speed domain and inducing results for wind power. This stochastic representation in the wind speed domain leads to complicated Markov chain models. The general results show that for an adequate wind modeling, higher order Markov chains should be used, combined with a high discretization of the wind speed domain (use of state width of $1 \mathrm{~m} / \mathrm{s}$ ). Past [8] and recent works [9] show results for the quality of wind speed simulation on different sampling time scales. Kaminsky et al. [10] give detailed information on the quality of different simulation methods, among them the MCMC, but due to the high resolution of the recorded time series (in the turbulence range), the application to wind power studies remains difficult to identify. In none of the mentioned papers, the impact of the wind turbines' power curves on the statistical relevance of the synthetic wind power time series is discussed.

\section{Markov Chain Monte Carlo Simulation}

\section{A. Definition of States of the Markov Chain}

Each process value is attributed to a state, defined based on the discretization of the recorded data. For example, an equal spacing of the states $\{1 \cdots m\}$ along the possible range of the values of the physical process $X$ is obtained by centering them at the state center vector $\zeta=\hat{X} / 2 m[2 k-1]$ with $k=1 \cdots m$, where $\hat{X}$ is the maximum process value. In the wind power domain, it is necessary to introduce two discrete states, namely $P \equiv 0$ and $P \equiv P_{N}$. The sequence of states $S_{t}$ is the result of such a discretization procedure.

\section{B. Markov Chain Basics}

1) First-Order Markov Chain: A stochastic process realization of the discrete-time r.v. $X$ taking discrete state values $S$ in the set $\{1 \cdots m\}$ is said to fulfill the hypothesis of a homogeneous first-order MC (FO-MC) if the process going from state $i$ to $j$ depends on no more than the state at $t=t-1$ and a conditional probability

$$
\operatorname{Pr}\left(X_{t}=j \mid X_{t-1}=i\right)=p_{i j}
$$

that is constant in time [11], [12]. The instants for the identification and definition of these conditional probabilities are equally spaced by a time step $\Delta$. It is then possible to formulate a $m \times m$ transition probability matrix $\mathbf{P}$ :

$$
\mathbf{P}=S_{t-1} \downarrow\left[\begin{array}{cccc}
p_{11} & p_{12} & \ldots & p_{1 m} \\
p_{21} & p_{22} & \ldots & p_{2 m} \\
\vdots & \vdots & \ddots & \vdots \\
p_{m 1} & p_{m 2} & \ldots & p_{m m}
\end{array}\right]
$$

According to this representation, each row of the matrix corresponds to the current state of the process, while each column to the possible next one, i.e., $p_{73}$ corresponds to the probability for the transition $7 \rightarrow 3$. The rows of the matrix sum up to 1 $\left(\sum_{j} p_{i j}=1\right)$ since this sum corresponds to the probability of a transition from a current state to any possible one. Concentration of probability masses along the main diagonal indicates a high tendency for chronological persistence of the Markov chain. The maximum likelihood estimate for the matrix entries is

$$
p_{i j}=\frac{n_{i j}}{\sum_{j} n_{i j}}
$$

with $n_{i j}$ being the number of transitions $i \rightarrow j$ encountered in the record.

The acf of an FO-Markov chain is described by an exponentially decaying function that can be analytically expressed once the transition probability matrix is known [13]. Fig. 2 shows that this assumption can be reasonable for our problem, ignoring at this point, the typical diurnal bumps due to the daily wind speed variation.

2) Higher Order Markov Chain: In cases when the present depends not only on the first lag but also on the last $n$ observations, an $n$-order Markov chain should be realized. In accordance to the FO-MC definition, the transition probabilities become:

$$
\operatorname{Pr}\left(X_{t}=j \mid X_{t-1}=i_{1}, \ldots, X_{t-n}=i_{n},\right)=p_{i_{n} i_{n-1} \ldots i_{1} j} .
$$

Each element $p_{i_{n} i_{n-1} \ldots i_{1} j}$ of $\mathbf{P}$ corresponds to the probability of the transition to state $j$ at $t=t$ when the system was at the states $i_{k}$ at $t=t-k, k \in[1, n]$. In the related literature, a cumbersome two-dimensional representation of $\mathbf{P}$ is proposed, as an extension of the FO-MC $\mathbf{P}$. As a more comprehensive representation, we propose a multidimensional $m^{n+1}$ transition probability matrix for the $n$ th-order MC, where each dimension corresponds to a previous state of the process, while the last dimension to the possible next one. For example, in a third-order $\mathrm{MC}$ (TO-MC), $p_{2473}$ is the probability for the transition to state 3 when the process has passed consecutively from states 2, 4, and 7. Consistent to the FO-MC $\mathbf{P}$, the elements of the last dimension of the matrix should sum up to $1\left(\sum_{j} p_{i_{n} i_{n-1} \ldots i_{1} j}=\right.$ $1)$. Thus, a high number of $\phi=m^{n}(m-1)$ independent 


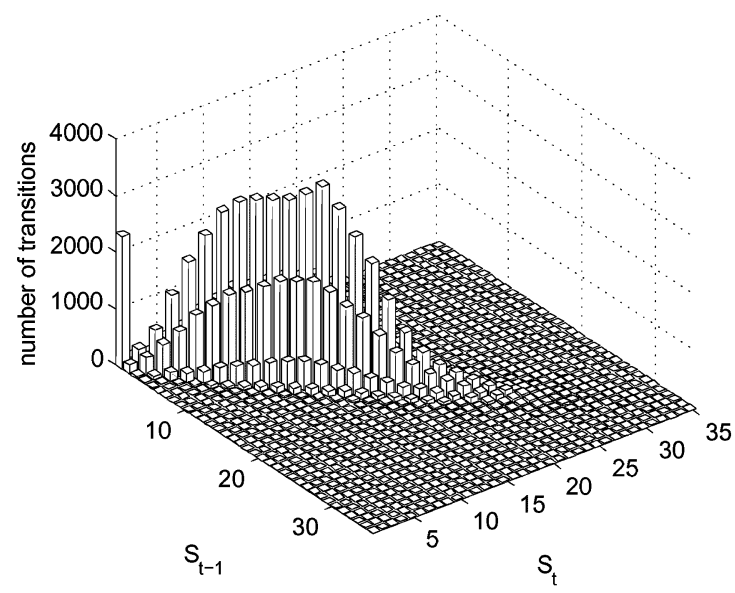

(a)

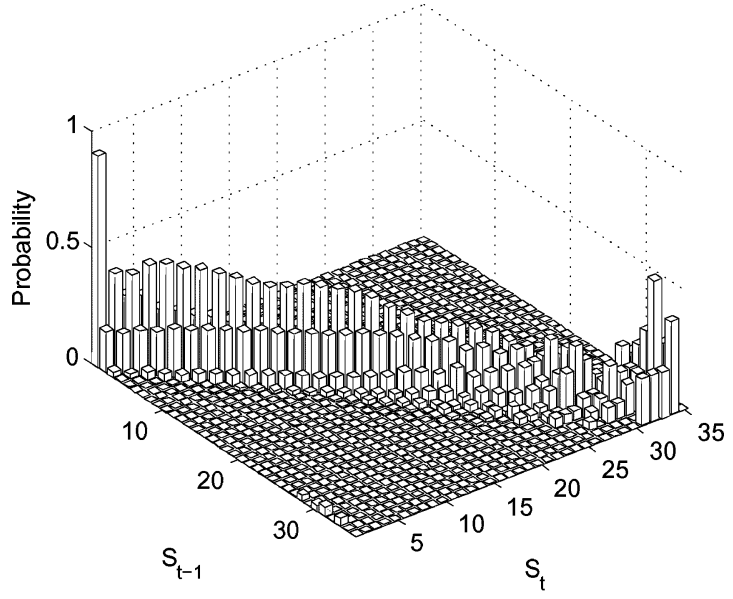

(b)

Fig. 3. Number of transitions and the respective estimation of $\mathbf{P}$ for the FO-MC. (a) Number of transitions. (b) Transition probabilities.

parameters must be estimated for the calculation of $\mathbf{P}$ [14], which is the main disadvantage that renders the applicability of the MCMC.

\section{MCMC Simulation}

Sampling based on a given transition probability matrix is performed by first constructing the cumulative probability transition matrix $\mathbf{P}_{\text {cum. }}$. In the case of an FO-MC, each row $i$ of $\mathbf{P}_{\text {cum }}$ corresponds to the discrete cumulative distribution function (cdf) $F_{i}$ for the next transition. In order to sample the next transition, a uniform random number $U$ is used. The realization $u$ of this random number is compared with elements of the $i$ th row of the matrix; if it falls between the elements $j-1$ and $j\left(F_{i(j-1)}<u \leq F_{i j}\right)$, the state $j$ is chosen as the next state. Further, the same procedure is repeated in order to sample based on the cdf $F_{j}$.

The multidimensional representation of $\mathbf{P}$ for higher order $\mathrm{MC}$ offers an easy implementation for the sampling algorithm for higher order MCMC. In this case, the discrete cdf for the next transition is obtained as the accumulation toward the last matrix dimension and is defined based on the $n$ previous states: $F_{i_{n} i_{n-i} \ldots i_{1}}$. Again, the same procedure is followed for the choice of the next state $j$, and the next cdf is obtained by shifting the indices one position leftwards: $F_{i_{n-i} \ldots i_{1 j}}$.

\section{Assessment of the Estimation Quality}

A typical procedure for the assessment of complex statistical models is the application of the Bayesian information criterion (BIC, see [15] and [16]). It is calculated by

$$
\mathrm{BIC}=\mathrm{LL}-\frac{\phi}{2} \ln \kappa
$$

where $\phi$ is the number of independent parameters [with $\left.\left.\max (\phi)=m^{n}(m-1)\right]\right), \kappa$ is the number of observations, and LL is the log-likelihood of the parameter estimates [17]:

$$
\mathrm{LL}=\sum_{i, j} n_{i j} \ln p_{i j} .
$$

Equation (6) gives one scalar for the estimation of the Markov chain; the model with a higher BIC is preferred. This formalism is a typical procedure for the assessment of the order of the MCMC models, since it penalizes the most complicated models, i.e., the ones with high number of independent parameters $\phi$. In [14], one may find the results for the application of the BIC to Markov chains of different orders.

\section{MARKov Chain DESCRIPTION OF WIND POWER}

As a numerical example, we perform the MCMC simulation in both the wind speed and the wind power domain and compare the pdf and acf of the original and the synthetic processes. The dataset used correspond to measurements of $10 \mathrm{~min}$ averages of wind speed for two years $(6 \times 24 \times 365 \times 2=105120$ data points, time series A (TS A) [18]). The maximum recorded wind speed is $34.4 \mathrm{~m} / \mathrm{s}$.

\section{A. MCMC on the Wind Speed Domain}

According to the general practice, the wind speeds are discretized into states of width $1 \mathrm{~m} / \mathrm{s}$, resulting in 35 states. The number of events contributing to the calculation of the entries of $\mathbf{P}$ range from 1 to 3491 (transition $13 \rightarrow 13$ ). Interesting for our purpose is the estimation quality: from the $m^{2}=1225$ possible transition events, only 430 take place in the dataset (35.1\%). This is mainly due to the chronological persistence of the process (most entries are gathered in the main diagonal). The same percentage for the second-order MC (SO-MC) is $5.7 \%$ (2445 transitions from a total of $35^{3}=42875$ ) while for the TO-MC, it drops to $0.5 \%$ (8432 transitions from a total of $\left.35^{4}=1500625\right)$.

Fig. 3(a) shows the number of counts for the estimation of $\mathbf{P}$, which is presented in Fig. 3(b). As may be seen, due to the impact of (4), entries in rows containing a low number of events will result to high probability entries in $\mathbf{P}$. Thus, in the formulation of $\mathbf{P}$, entries with low information will be treated beneficially. The application of higher order MC models magnifies this problem, since the available transitions are scattered in a larger space. 


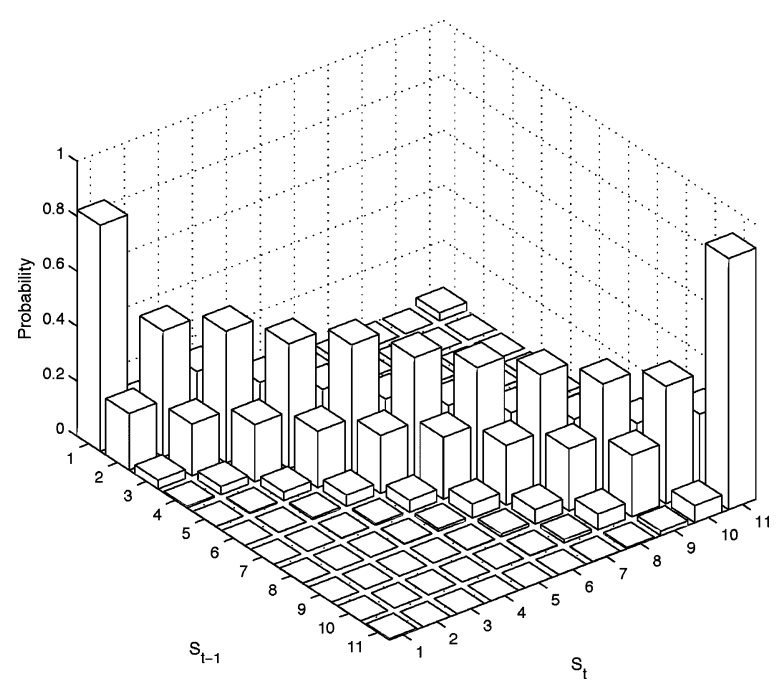

Fig. 5. Estimation of the FO-MC $\mathbf{P}$ in the wind power domain.

The low-information high-probability entries in $\mathbf{P}$ for SO- and TO-MC will be significantly increased. This is a basic problem that renders the applicability of higher order MC models: the more states used for the representation of the initial process, the more cases of low information encountered in the model, leading to a poorer model quality. A major improvement of the MC model may be achieved by the reduction of the number of states; in the following section, we show how this reduction is performed by working in the wind power domain.

\section{B. MCMC on the Wind Power Domain}

The state reduction in the wind power domain is presented in Fig. 4. As mentioned, due to the transformation shown in Fig. 1, the wind speed range is divided into three areas presented in (1). The regions A and B in the wind power domain correspond to two distinct states. By using the same number of states in the wind speed and power domain for the variable power range (area C - nine states), we end up with a state reduction from 35 to 11 states. Thus, by estimating $\mathbf{P}$ after the transformation of the wind speed through the wind turbine curve, we achieve a significant reduction in the number of states without losing any information. The number of independent parameters is, therefore, significantly reduced to 110 from 1190 for the FO-MC, to 1210 from 41650 for the SO-MC, and to 13310 from 1457750 for the TO-MC. The estimated wind power transition matrix $\mathbf{P}$ is illustrated in Fig. 5. In this case, the matrix entries show a significantly lower uncertainty than in the first case.

Together with the state reduction, the transition to the wind power domain leads to a general simplification of the stochastic model. As shown in Fig. 2, wind power time series show a lower persistence than the wind speed ones. This means that the same stochastic model in the wind power domain is achieved by a lower order Markov chain. This result is verified by the use of the BIC; in particular, while in the wind speed domain, the BIC shows a much higher accuracy for the TO-MC model, in the wind power domain, all three MC models (FO-MC, SO-MC, and TO-MC) provide the same level of accuracy.

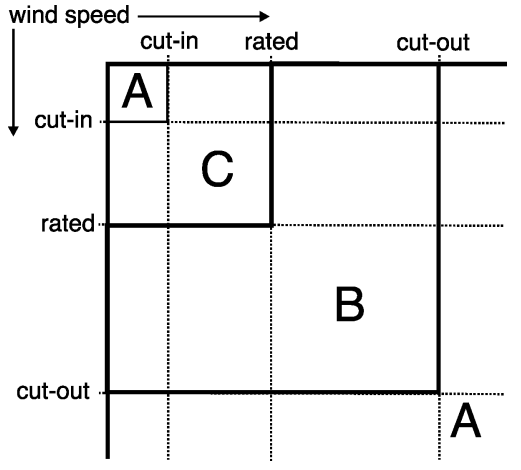

Fig. 4. Division of the wind domain into lumped states.

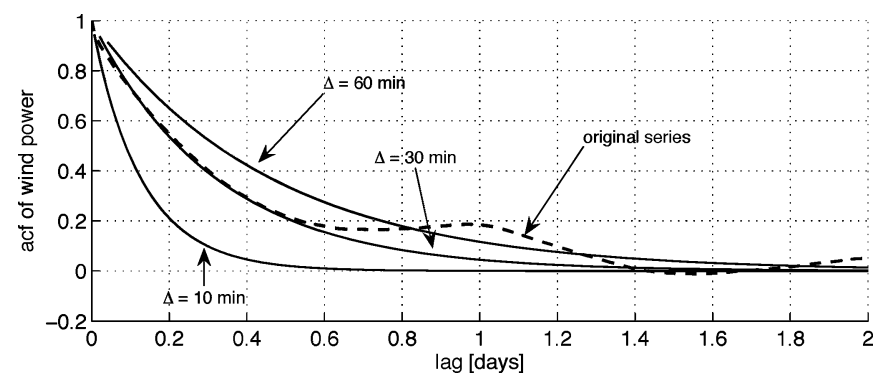

Fig. 6. Comparison of the FO-MC acf reconstruction for different averaging windows for wind power. The best result in this example can be achieved for $\Delta=30 \min (\mathrm{TS} \mathrm{A})$.

\section{Influence of the Sampling Frequency}

The FO-MC, however, does not always serve for a satisfactory reproduction of the acf. This is the case for highly volatile signals, where the lag-1 time step is only "part of the truth" about the persistence of the time series. We encountered this behavior in most recorded wind speed and wind power time series with a time step below $30 \mathrm{~min}$, and therefore, propose the concept of customized averaging. For a detailed exposition of the implications of wind speed averaging on some statistical parameters, see [19].

The original time series can be manipulated by

$$
X_{t, \text { new }}=\sum_{\alpha} X_{t-\alpha \delta} / \alpha
$$

where $\alpha \delta=\Delta$ and $\delta$ is the step in the original series, e.g., 10 min. Our results indicate that for the FO-MC, a typical averaging window of $\Delta=1 \mathrm{~h}$ is necessary for a satisfactory reconstruction of the wind power acf from the wind speed time series (method 1), while for the pretransformed wind power time series (method 2), $\Delta=0.5 \mathrm{~h}$ is the best approximation. Fig. 6 shows an example.

For different values of $\Delta$, one will generally get different results for the best order of the Markov chain to fit the model. For example, for the data in TS A, we get the results for the optimal order as listed in Table I. We verified this result also using measurement data with lower frequency. In particular, a dataset consisting of 20 years of measurements of $1 \mathrm{~h}$ averages of wind speed for several locations in The Netherlands (TS B) [20] were used. In this case, an FO-MC was identified to fit the time-series data. 
TABLE I

ESTIMATED ORder OF WIND POWER MODEL From TS A

\begin{tabular}{c||c|c|c|c|c}
\hline \multicolumn{1}{c||}{} & \multicolumn{5}{c}{ number of states $m$} \\
\hline$\Delta$ & 10 & 15 & 20 & 25 & 30 \\
\hline $10 \mathrm{~min}$ & 3 & 1 & 1 & 3 & 3 \\
\hline $20 \mathrm{~min}$ & 2 & 1 & 3 & 3 & 3 \\
\hline $30 \mathrm{~min}$ & 1 & 1 & 3 & 3 & 3 \\
\hline $60 \mathrm{~min}$ & 1 & 1 & 3 & 3 & 3 \\
\hline
\end{tabular}

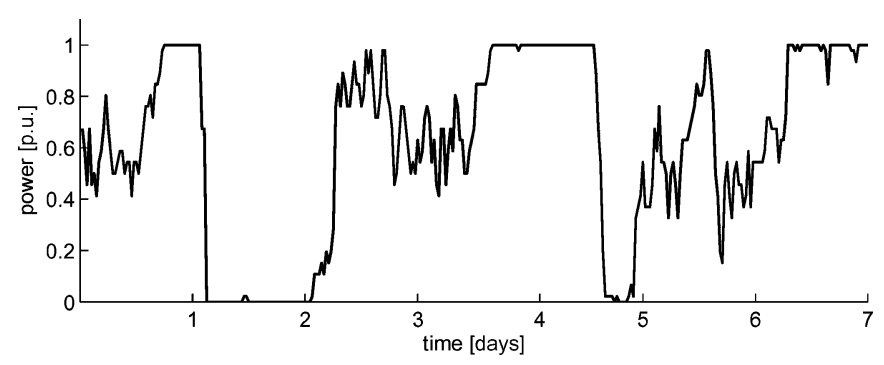

Fig. 7. Simulation of wind power based on an TO-MCMC. The persistence of the signal is well visible.

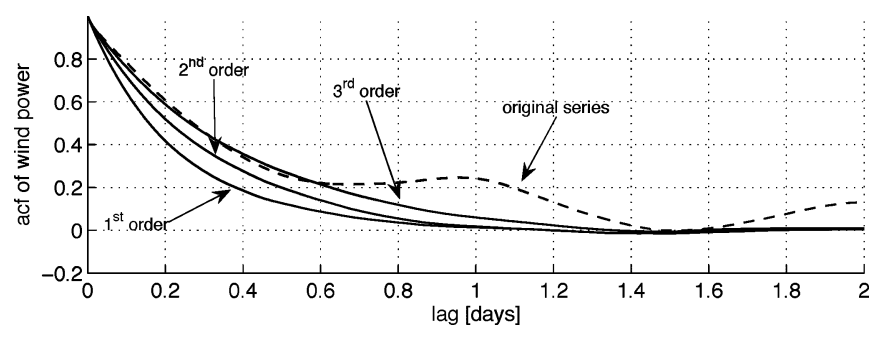

Fig. 8. Simulation results for the acf of $10 \mathrm{~min}$ MC models of different orders for TS A.

Note that the optimal order is typically estimated too high for very short measured time series; therefore, this criterion can only serve as an additional source of information in addition to visual inspection of simulated pdf and acf. In Table I, the estimated order of the wind power model of TS A is presented. We can see that for this time series, one can only identify the general trend that for a low number of states, the order goes down as the averaging time goes up, while for high number of states, the BIC will tend to choose the least parsimonious model.

Thus, the state reduction in the power domain directly serves for an improvement of the estimated statistical model. Independent from the sampling frequency of the measured data, simpler MC models may be used for the modeling of the wind power output in the wind power domain.

\section{Simulation Results}

For verification, we generated a synthetic wind power time series based on TS A, for MC models of different orders, in the wind power domain. The length of the simulated time series was chosen to be twice the length of the original one (four years). In Fig. 7, we present the simulated wind power output for one week based on an TO-MCMC. The persistence of the simulated signal is well visible. We can, for example, verify the result that the optimum order $n$ is 3 for $m=25$ by visual inspection of the acf in Fig. 8. Also, the fit for the pdf is absolutely satisfactory (see Fig. 9). We may, therefore, conclude that the method is
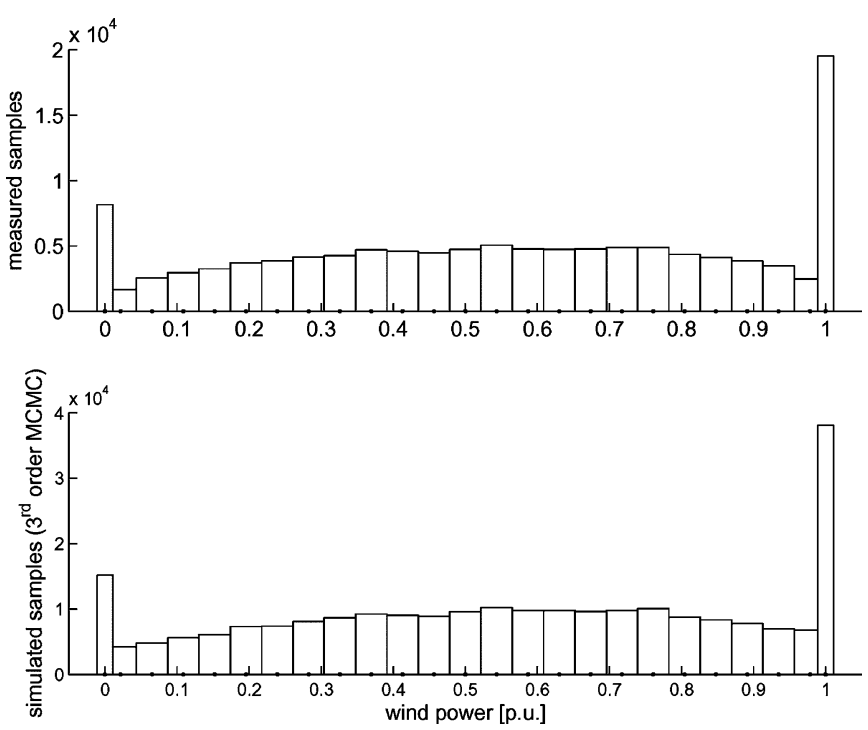

Fig. 9. Comparison of the pdf of the original TS A (above) and a simulated TO version of double length (below). The pdf is well approximated by the simulation.

suitable for reproducing the two basic statistical wind power parameters with sufficient accuracy.

The same analysis was performed for TS B $(\delta=1 \mathrm{~h})$. We inspected in a similar manner and identified excellent agreement in the pdf and acf by employing an FO-MC without further averaging.

\section{CONCLUSION-FUTURE WORK}

In this contribution, it is shown that the direct generation of wind power time series based on the MCMC is superior to indirect synthesization of wind power via artificial wind speeds. The MCMC is the method that provides results consistent to the nonstandard pdf and the respective acf for the modeling of the stochastic output of the wind plant.

One of the main advantages of the method is that this shift of the modeling process to the wind power domain enables the treatment of recorded wind power data as source for the model recognition. Future extensions of this paper will directly treat such data instead of wind speed data as the input for the identification process. This straightforward approach is the main step toward the development of wind turbine/wind park black-box models, since during the modeling process, the definition of the cumbersome transformation $v \rightarrow P$ in cases of increasing modeling depth of single or aggregated generation units is avoided.

\section{ACKNOWLEDGMENT}

This work is the result of an ongoing collaboration between the authors' institutes.

\section{REFERENCES}

[1] B. Klöckl, "Impacts of energy storage on power systems with stochastic generation," Ph.D. dissertation, Swiss Federal Inst. Technol. (ETH), Zurich, Switzerland, Hartung-Gorre Verlag, 2007, (ISBN978-3-86628190-5). 
[2] S. Klein and W. Beckman, "Loss-of-load probabilities for stand-alone photovoltaic systems," Solar Energy, vol. 39, no. 6, pp. 499-512, 1987.

[3] G. Papaefthymiou, P. H. Schavemaker, V. der Sluis, W. L. Kling, D. Kurowicka, and R. M. Cooke, "Integration of stochastic generation in power systems," (Invited Paper), presented at the 15th Power Syst. Comput. Conf. PSCC 2005, Liege, Belgium, Aug. 22-26.

[4] J. Hennessey, "Some aspects of wind power statistics," J. Appl. Meteorol., vol. 16, no. 2, pp. 119-128, 1977.

[5] G. Box and G. Jenkins, Time Series Analysis-Forecasting and Control. San Francisco, CA: Holden-Day, 1976.

[6] B. Brown, R. Katz, and A. Murphy, "Time series models to simulate and forecast wind speed and wind power," J. Climate Appl. Meteorol., vol. 23, pp. 1184-1195, Aug. 1984.

[7] M. Blanchard and G. Desrochers, "Generation of autocorrelated wind speeds for wind energy conversion system studies," Solar Energy, vol. 33, no. 6, pp. 571-576, 1984.

[8] D. Jones and M. Lorenz, "An application of a Markov chain noise model to wind generator simulation,” Math. Comput. Simul., vol. 28, pp. 391-402, 1986.

[9] A. Shamshad, M. A Bawadi, W. Hussin, T. Majid, and S. Sanusi, "First and second order Markov chain model for synthetic generation of wind speed time series," Energy, vol. 30, pp. 693-708, 2005.

[10] F. Kaminsky, R. Kirchhoff, C. Syu, and J. Manwell, "A comparison of alternative approaches for the synthetic generation of wind speed time series," J. Solar Energy Eng., vol. 113, pp. 280-289, 1991.

[11] L. Breiman, Probability and Stochastic Processes-With a View Toward Applications. Boston, MA: Houghton Mifflin, 1969.

[12] A. Papoulis, Probability, Random Variables, and Stochastic Processes. New York: McGraw-Hill, 1984

[13] I. Basawa, "Estimation of the autocorrelation coefficient in simple Markov chains," Biometrika, vol. 59, no. 1, pp. 85-89, 1972.

[14] A. Berchtold and A. Raftery, "The mixture transition distribution model for high-order Markov chains and non-gaussian time series," Stat. Sci., vol. 17 , no. 3 , pp. $328-356,2002$.

[15] G. Schwarz, "Estimating the order of a model," Ann. Stat., vol. 6, no. 2, pp. 461-464, 1978.

[16] R. Katz, "On some criteria for estimating the order of a Markov chain," Technometrics, vol. 23, no. 3, pp. 243-249, Aug. 1981.

[17] J. Gani, "Some theorems and sufficiency conditions for the maximumlikelihood estimator of an unknown parameter in a simple Markov chain," Biometrika, vol. 42, no. 3/4, pp. 342-359, 1955.
[18] Center for Energy. Efficiency \& Renewable Energy. (2005). [Online]. Available: http://www.ceere.org.

[19] X. G. Larsén and J. Mann, "The effects of disjunct sampling and averaging time on maximum mean wind speeds," J. Wind Eng. Ind. Aerodyn., vol. 94, no. 8, pp 581-602, Aug. 2006.

[20] Hydra project: Wind climate assessment of The Netherlands. (2005). [Online]. Available: http://www.knmi.nl/samenw/hydra

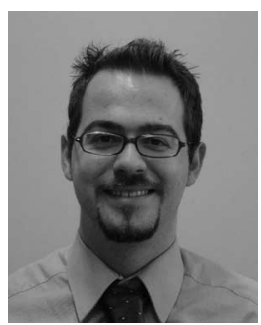

George Papaefthymiou (M'03) received the Dipl.Eng. degree in electrical and computer engineering from the University of Patras, Patras, Greece, in 1999, and the Ph.D. degree from Delft University of Technology, Delft, The Netherlands, in 2007.

$\mathrm{He}$ is currently with the Electrical Power systems Laboratory, Delft University of Technology as a Research Associate. His current research interests include modeling of uncertainty in power systems and design of systems with large-scale penetration of distributed and stochastic generation. He is involved in the main framework of the intelligent power systems.

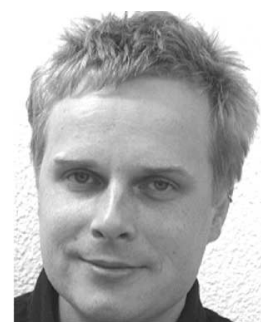

Bernd Klöckl (M'02) received the M.Sc. degree in electrical power engineering from Graz University of Technology, Graz, Austria, in 2001, and the Ph.D. degree from Swiss Federal Institute of Technology, Zurich, Switzerland in 2007.

From 2002 to 2006, he was a Research Associate at the High Voltage Laboratory, Swiss Federal Institute of Technology (ETH), Zurich. From 2006 to 2007, he has been the Head of the Power Grid Unit, Austrian Association of Electricity Companies, Vienna, Austria. Since 2007, he has been with the Market Management Unit of the Austrian transmission system operator, Verbund Austrian Power Grid, Vienna, Austria. His current research interests include energy storage theory and the related stochastic power generation issues.

Mr. Klöckl is member of Austrian Engineering Society (OVE) and International Conference on Large Electric High-Tension Systems (CIGRÉ). 